\title{
In silico profiling of non-synonymous SNPs in IDS gene for early diagnosis of Hunter syndrome
}

\author{
Adarshan Sivakumar ${ }^{1}$, Yuvaraj Dinakarkumar ${ }^{3}$, Wahidah H. Al-Qahtani ${ }^{2}$, Muthusamy Karnan ${ }^{4}$, \\ Jothiramalingam Rajabathar ${ }^{2}$, Arokiyaraj Charumathi ${ }^{5}$, Elakiya Sadhaasivam ${ }^{6}$, Aparna Preetha Venugopal ${ }^{7}$ \\ Baljeet Mukhtiar Singh ${ }^{8}$, Maqbool Qutub ${ }^{9}$ and Sai Ramesh Anjaneyulu ${ }^{3^{*}}$ (])
}

\begin{abstract}
Background: Single amino acid substitutions in the Iduronate-2-sulfatase enzyme result in destabilization of the protein and cause a genetic disorder called Hunter syndrome. To gain functional insight into the mutations causing Hunter syndrome, various bioinformatics tools were employed, and special significance is given to molecular docking.

Results: In-silico tools available online for preliminary analysis including SIFT, PolyPhen 2.0, etc., were primarily employed and have identified 51 Non-synonymous Single Nucleotide Polymorphisms (ns-SNPs) as possibly deleterious. Further, modelling and energy minimization followed by Root Mean Square Deviation (RMSD) calculation has labelled 42 mutations as probably deleterious ns-SNPs. Later, trajectory analysis was performed using online tools like PSIPRED, SRide, etc., and has predicted six ns-SNPs as potentially deleterious. Additionally, docking was performed, and three candidate ns-SNPs were identified. Finally, these three ns-SNPs were confirmed to play a significant role in causing syndrome through root mean square fluctuation (RMSF) calculations.

Conclusion: From the observed results, G134E, V503D, and E521D were predicted to be candidate ns-SNPs in comparison with other in-silico tools and confirmed by RMSF calculations. Thus, the identified candidate ns-SNPs can be employed as a potential genetic marker in the early diagnosis of Hunter syndrome after clinical validation.
\end{abstract}

Keywords: IDS gene, Hunter syndrome, Molecular docking, Trajectory analysis, RMSF, Ns-SNPs

\section{Introduction}

Hunter syndrome, a noxious and uncommon lysosomal storage disease, which is $\mathrm{X}$-linked and caused by the mutation within the IDS gene [1], coding a lysosomal polypeptide of 550 amino acid length (IDS, E.C. 3.1.6.13) [2], located at chromosome Xq27.3-q28, spans $24 \mathrm{~Kb}$, composed of 9 exons and belongs to the sulfatase family [3]. It is also known as type II Mucopolysaccharidosis (OMIM 309900). IDS gene codes for the significant

\footnotetext{
*Correspondence: drsairamesh@gmail.com

3 Department of Biotechnology, Vel Tech High-Tech Dr. Rangarajan Dr. Sakunthala Engineering College, Avadi-Vel Tech Road, Chennai, Tamil Nadu, India

Full list of author information is available at the end of the article
}

enzyme that prevents the build-up of glycosaminoglycans (GAGs) in the lysosome.

IDS breaks-down heparan sulfate (HS) and dermatan sulfate (DS) by specifically targeting the sulfate group at the O-2 position [4]. This enzyme contains 25 amino acid residues at the amino-terminal, that acts as a signal peptide followed by 8 amino acids. These 33 amino acids are cleaved after processing and thus the functional length of the enzyme is 517 amino acids.

Activity of IDS gene is crucial for cellular maintenance [5]. Hence, defect in this gene leads to the buildup of GAGs - HS and DS in the body. It is estimated that Hunter syndrome occurs in approximately one in 170,000 male live births and prevails in individuals of all 
ethnicities [6,7]. More than 500 disease-causing mutations have been identified in the IDS gene, which include single nucleotide polymorphisms (SNPs) that influence the structure and function of the enzyme greatly. Thus, SNPs can be considered as a diagnostic marker for Hunter syndrome [8].

People with Hunter syndrome show various clinical manifestations such as respiratory infections [9], sleep apnea, joint stiffness, pelvic dysplasia, hepatomegaly, umbilical and inguinal hernias, otitis, gingival hypertrophy, hyperplasia [10], cardiological and ocular manifestations, and also skin infections such as Mangolian spots and lesions [11]. Due to the non-availability of the drug, patients are advised for enzyme replacement therapy (ERT) or stem cell therapy (SCT) $[12,13]$.

The current study utilized a combination of bioinformatic tools to predict the structural and functional modifications of proteins and aims to identify candidate non-synonymous single nucleotide polymorphisms (nsSNPs) in the IDS gene that may be used for specific and appropriate attuned therapies for Hunter syndrome in near future.

\section{Methodology}

\section{Datasets and retrieval of nsSNPs}

National Centre for Biotechnology Information (NCBI) (https://www.ncbi.nlm.nih.gov/snp/) is an open-source database [14], from which the non-synonymous (missense) SNPs in the IDS gene of Homo sapiens and protein sequence in FASTA format were retrieved. Recovered SNPs were subjected to look into their deleterious effect on the IDS gene by employing a set of insilico tools.

\section{Combined prediction using preliminary tools}

FASTA format of the IDS gene was given as input in tools as shown in Table 1 and ns-SNPs predicted as deleterious by all 5 tools were considered for further analysis.

\section{Modelling and RMSD calculation}

The three-dimensional structure has a momentous role in analysing the trajectory of the protein. Since no structure was available for the protein IDS, a 3-D structure was modelled through SWISS-MODEL expasy (http://swiss model.expasy.org/) [20], validated using PROCHECK [21] and ProSA Webserver (https://prosa.services.came. sbg.ac.at/prosa.php) [22]. In due course, all mutant structures were generated using SWISS-PDB Viewer [23]. Once generated, all the structures including native were energy minimized using GROMACS, which works on 1-bfgs (imited-memory Broyden-Fletcher-GoldfarbShanno quasi-Newtonian minimizer) [24]. Structures obtained from GROMACS were utilized for root mean square deviation (RMSD) calculation [25] using SWISSPDB Viewer.

\section{Trajectory analysis}

IDS gene, either as a sequence or as a structure file was submitted in tools as shown in Table 2 for trajectory analysis. ns-SNPs predicted as deleterious in the maximum number of tools were selected for further analysis.

Table 2 List of tools employed for trajectory analysis

\begin{tabular}{lll}
\hline S. no. & Trajectory tool & Prediction \\
\hline 1 & $\begin{array}{l}\text { PSIPRED } \\
\text { (http://bioinf.cs.ucl.ac.uk/psipred/) [26] }\end{array}$ & Secondary structure \\
2 & $\begin{array}{l}\text { SRide } \\
\text { (http://sride.enzim.hu/) [27] } \\
\text { Consurf } \\
\text { (https://consurf.tau.ac.il/) [28] }\end{array}$ & Stability residues \\
4 & $\begin{array}{l}\text { Netsurf } \\
\text { (http://www.cbs.dtu.dk) [29] }\end{array}$ & Conserved residues \\
5 & $\begin{array}{l}\text { PredyFlexy } \\
\text { (https://www.dsimb.in) [30] }\end{array}$ & RSA \\
6 & $\begin{array}{l}\text { Missense 3D } \\
\text { (http://www.sbg.bio.ic.ac.uk/ misse } \\
\text { nse3d/) [31] }\end{array}$ & Flexibility of residues \\
& & Buried charge switch
\end{tabular}

Table 1 List of tools employed for combined analysis using preliminary tools

\begin{tabular}{|c|c|c|}
\hline S. no. & Preliminary tools & Prediction mechanism \\
\hline 1 & $\begin{array}{l}\text { SIFT } \\
\text { (https://sift.bii.a-star.edu.sg/www/SIFT_seq_submit2.html) [15] }\end{array}$ & Sequence-homology \\
\hline 2 & $\begin{array}{l}\text { PolyPhen } 2.0 \\
\text { (http://genetics.bwh.harvard.edu/pph2/) [16] }\end{array}$ & Structure-homology \\
\hline 3 & $\begin{array}{l}\text { PANTHER } \\
\text { (http://www.pantherdb.org/tools/csnpScore.do) [17] }\end{array}$ & Hidden Markov model \\
\hline 4 & $\begin{array}{l}\text { I-Mutant } 3.0 \\
\text { (http://gpcr2.biocomp.unibo.it/cgi/predictors/I-Mutant3.0/I-Mutant3.0.cgi) [18] }\end{array}$ & Support vector machine \\
\hline 5 & $\begin{array}{l}\text { PMut } \\
\text { (https://mmb.irbbarcelona.org/PMut/pymut) [19] }\end{array}$ & Neural-network \\
\hline
\end{tabular}




\section{Molecular docking and visualization}

Docking was performed by using Autodock Vina [32]. The results were visualized and analyzed using Ligplot+ [33].

\section{RMSF calculation}

Root mean square fluctuation (RMSF) was calculated using CABS-flex 2.0 server (http://biocomp.chem.uw. edu.pl/FCABSflex2) [34].

\section{Effect of SNP on splicing}

Splicing is the most significant post-transcriptional modification, which removes the non-coding regions and collate the exons to form the functional protein. To evaluate the influence of candidate SNPs on the splicing sites, NetGene 2 online server (https://services. healthtech.dtu.dk/service.php?NetGene2-2.42) was employed. This server was provided with the DNA sequence in FASTA format as input.

\section{Pathogenicity analysis}

At last, all the candidate SNPs were employed for pathogenicity analysis to confirm and further support the prediction. This analysis was performed using DUET online server (http://biosig.unimelb.edu.au/duet/) [36].

\section{Results and discussion}

\section{Datasets and retrieval of ns-SNPs}

The IDS gene contains 5549 SNPs in total (As of 22nd September 2020). Out of these 5549 SNPs, only 289 non-synonymous SNPs (missense mutation) were retrieved from the National Centre for Biotechnology Information (NCBI) database. Missense mutation contributes $5 \%$ among total SNPs in the IDS gene $(289 / 5549)$.

\section{Combined analysis using preliminary tools}

The combined analysis of five online tools had predicted 51 out of 289 ns-SNPs as deleterious in common and the individual prediction by the tools are listed in Table 3. Since the preliminary tools involve different algorithms, a combined approach may increase the precision in identifying the deleterious ns-SNPs [37]. Only the predicted 51 possibly deleterious ns-SNPs were considered for the subsequent analysis.

\section{Modelling and RMSD calculation}

A three-Dimensional structure is required for understanding the structure-function relationship of proteins, since point mutation can drastically affect protein function [38]. The 3D structure of IDS protein
Table 3 Results of combined prediction using preliminary analysis

\begin{tabular}{llll}
\hline S. no. & Tools & SNPs analysed & SNPs selected \\
\hline 1 & SIFT & 289 & 124 \\
2 & PolyPhen 2.0 & 124 & 57 \\
3 & PANTHER & 57 & 54 \\
4 & I-Mutant 3.0 & 54 & 52 \\
5 & PMut & 52 & 51 \\
\hline
\end{tabular}

modelled using SWISS-MODEL was based on the template 5fql.1.A (99.81\% similarity) and with the QMEAN score of -1.21 .

The structure was further validated using PROCHECK and ProSA webserver. Ramachandran Plot shows the presence of $86.5 \%$ of residues within the most favourable regions, which indicates the structure obtained is in good quality [39] and the overall z-score for the protein IDS was found to be -8.14 using the ProSA server.

Since the structure obtained is a homodimer, only the Chain A was used to generate the 51 mutant structures using SWISS-PDB viewer.

Table 4 List of ns-SNPs predicted as deleterious through RMSD calculation through GROMACS

\begin{tabular}{llllll}
\hline S.no. & $\begin{array}{l}\text { Residual } \\
\text { change }\end{array}$ & RMSD (Å) & S.no. & $\begin{array}{l}\text { Residual } \\
\text { change }\end{array}$ & RMSD (Å) \\
\hline 1 & D45H & 0.245 & 22 & C422Y & 0.475 \\
2 & S61P & 0.226 & 23 & Q465K & 0.174 \\
3 & A79G & 0.327 & 24 & Y466C & 0.202 \\
4 & V83G & 0.354 & 25 & P467L & 0.218 \\
5 & P86L & 0.502 & 26 & R468P & 0.625 \\
6 & R88C & 0.196 & 27 & R468L & 0.216 \\
7 & P97T & 0.264 & 28 & R468W & 0.161 \\
8 & P120H & 0.178 & 29 & D478G & 0.197 \\
9 & T130I & 0.169 & 30 & P480S & 0.187 \\
10 & G134E & 0.237 & 31 & M488I & 0.235 \\
11 & A205P & 0.401 & 32 & G489A & 0.43 \\
12 & H229R & 0.198 & 33 & R493H & 0.165 \\
13 & D252Y & 0.245 & 34 & R493C & 0.242 \\
14 & R271W & 0.257 & 35 & Y497C & 0.203 \\
15 & R297C & 0.299 & 36 & Y497N & 0.222 \\
16 & G312D & 0.449 & 37 & R498T & 0.183 \\
17 & G312S & 0.292 & 38 & T500I & 0.197 \\
18 & G336W & 0.165 & 39 & W502S & 0.281 \\
19 & L339P & 0.46 & 40 & V503D & 0.321 \\
20 & P358T & 0.282 & 41 & E521D & 0.189 \\
21 & T409M & 0.268 & 42 & D532G & 0.341 \\
\hline & & & & &
\end{tabular}


Table 5 Results of trajectory analysis

\begin{tabular}{|c|c|c|c|c|c|c|c|c|}
\hline S. no. & Tools & Protein & G134E & P358T & R468W & M488I & V503D & E521D \\
\hline \multirow[t]{2}{*}{1} & \multirow[t]{2}{*}{ Psipred } & Native & $C$ & C & C & $C$ & $\mathrm{E}$ & E \\
\hline & & Mutant & $E$ & E & $C$ & $E$ & C & C \\
\hline \multirow[t]{2}{*}{2} & \multirow[t]{2}{*}{ SRide } & Native & $\$$ & & & & & \\
\hline & & Mutant & $\$, \#$ & $\$, \#$ & $\$, \#$ & $\$, \#$ & $\$, \#$, D334 & $\$, \#$ \\
\hline \multirow[t]{2}{*}{3} & \multirow[t]{2}{*}{ Consurf } & Native & 6 & 8 & 6 & 9 & 9 & 8 \\
\hline & & Mutant & 4 & 7 & 5 & 8 & 9 & 7 \\
\hline \multirow[t]{2}{*}{4} & \multirow[t]{2}{*}{ Netsurf } & Native & $1 \%$ & $1 \%$ & $26 \%$ & $10 \%$ & $7 \%$ & $26 \%$ \\
\hline & & Mutant & $2 \%$ & $2 \%$ & $18 \%$ & $10 \%$ & $18 \%$ & $18 \%$ \\
\hline \multirow[t]{2}{*}{5} & \multirow[t]{2}{*}{ Predyflexy } & Native & 1 & 0 & 1 & 2 & 0 & 1 \\
\hline & & Mutant & 1 & 0 & 2 & 1 & 0 & 0 \\
\hline \multirow[t]{2}{*}{6} & \multirow{2}{*}{$\begin{array}{l}\text { Missense } \\
3 \mathrm{D}\end{array}$} & Native & Uncharge & No change & Charge & No change & Uncharge & No change \\
\hline & & Mutant & Charge & & Uncharge & & Charge & \\
\hline
\end{tabular}

: D45, V133, P358; \#:V233

Table 6 Tabulation of Binding energies in Kcal/mol found using Autodock vina

\begin{tabular}{llllllll}
\hline & Native & G134E & P358T & R468W & M488I & V503D & E521D \\
\hline DS & -6.6 & -6.6 & -6.6 & -6.6 & -6.6 & -6.6 & -6.6 \\
HS & -6.3 & -6.0 & -6.6 & -6.3 & -6.6 & -5.9 & -5.6 \\
LS & -8.6 & -8.5 & -6.8 & -8.6 & -6.8 & -8.6 & -8.6 \\
LS+HS & -6.3 & -6.3 & -7.0 & -5.6 & -6.9 & -6.3 & -7.0 \\
LS+DS & -6.6 & -6.6 & -7.1 & -6.6 & -7.1 & -6.6 & -6.6 \\
\hline
\end{tabular}

Ultimately, Energy minimization was done for all structures including native using GROMACS, and RMSD values were calculated by super-imposing native and mutant structures in SWISS-PDB Viewer. Based on the RMSD values of 51 SNPs, the threshold was fixed as above $0.15 \AA$ (>0.15 $\AA$ ). Over one-third of the mutants have RMSD value greater than 0.15 , the higher the RMSD value, the higher the degree of deleterious effect [40]. Based on RMSD values, 42 SNPs were found to be probably deleterious and are enlisted in Table 4.

\section{Trajectory analysis}

Only the possibly deleterious ns-SNPs (42 ns-SNPs) were analysed using trajectory tools. The analysis had predicted 6 ns-SNPs (G134E, P358T, R468W, M488I, V503D, and E521D) as potentially deleterious in common by maximum number of tools (Table 5). Multiple approaches were employed for trajectory analysis using various tools, to get more accurate results. Among these ns-SNPs five SNPs (G134E, P358T, M488I, V503D, and E521D) have variations in their secondary structures after mutation and all these 6 SNPs have modified stability residues in comparison with the native structures. The conservation score also varies significantly along with the Relative Solvent Accessibility values. Analogously, a study has been communicated, which also employs these properties for the prediction of most deleterious mutation in $H B A 1$ gene [39].

\section{Molecular docking and visualization}

Protein-Ligand Docking Analysis demonstrated that the mutant structures bind to the ligand in a slightly different orientation compared to the native protein. Three ligands were used for docking within which two were the enzyme's own substrates (dermatan sulfate, DS and heparan sulfate, HS) and one from a plant source (Luteolin Sulfate -LS). LS was selected as a ligand because of its abundant availability [41] and its structural similarity with HS and DS. The binding energy of the ligands with mutants were compared with native protein.

InterPro server [42] and literature survey [5] identified D45, D46, C84, R88, K135, F137, H138, H229, D334, $\mathrm{H} 335$, and $\mathrm{K} 347$ (11 residues) as active site residues, and site-specific docking was performed using Autodock Vina. From the results of docking, it is obvious that binding energy differs drastically in the case of G134E, V503D, and E521D (especially for the ligand-Heparan 


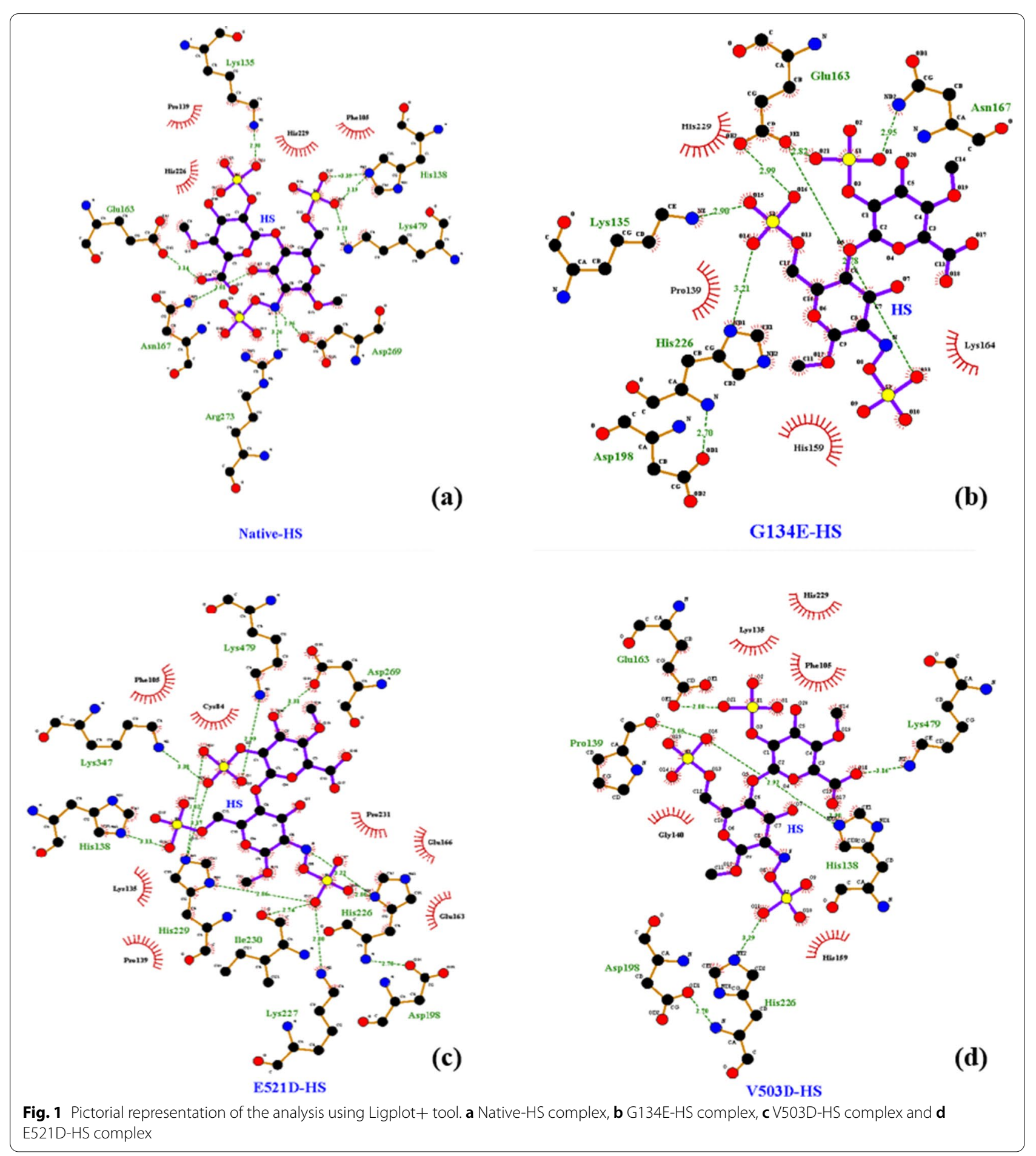


Table 7 RMSF values of potentially deleterious SNPS

\begin{tabular}{llll}
\hline S. no. & SNPs & Native & Mutant \\
\hline 1 & G134E & 0.529 & 0.227 \\
2 & V503D & 0.362 & 0.171 \\
3 & E521D & 0.593 & 0.121 \\
\hline
\end{tabular}

Sulfate) (Table 6). Binding energies for remaining residues vary very slightly. Thus, HS was hypothesised to play a crucial role in causing Hunter's syndrome and these three ns-SNPs were considered as candidate nsSNPs. Binding energy of these mutant proteins with HS in the presence of LS were calculated (Table 6). From the results, it is observed that binding of LS with the protein alters the protein structure and increases the affinity for the ligand. Thus, LS may act as a potential molecule in treating Hunter Syndrome.

The interaction between native and mutant proteins (G134E, V503D, and E521D) with the ligand Heparan
Table 8 Results of pathogenicity analysis using DUET web server

\begin{tabular}{|c|c|c|c|c|}
\hline \multirow[t]{2}{*}{ SNPs } & \multicolumn{4}{|l|}{ DUET } \\
\hline & $\begin{array}{l}\operatorname{mCSM}(\Delta \Delta G) \\
(\mathrm{kcal} / \mathrm{mol})\end{array}$ & $\begin{array}{l}\operatorname{SDM}(\Delta \Delta \mathrm{G}) \\
(\mathrm{kcal} / \mathrm{mol})\end{array}$ & $\begin{array}{l}\text { DUET }(\Delta \Delta \mathrm{G}) \\
(\mathrm{kcal} / \mathrm{mol})\end{array}$ & Prediction \\
\hline G134E & -2.378 & -3.07 & -2.706 & Destabilizing \\
\hline V503D & -3.276 & -3.34 & -3.64 & Destabilizing \\
\hline E521D & -1.838 & -1.45 & -1.952 & Destabilizing \\
\hline
\end{tabular}

Sulfate were visualized and bonds formed in native and mutants were observed using ligplot+ (Fig. 1). Ligplot+ analysis shows the variations in bonds formed by mutant proteins with ligands, compared with native protein. The mutation could also be the rational for these variations [43] and it supports the conclusions obtained from all the previous analyses. These predictions are in accordance with the deviation in RMSD values, as predicted earlier.

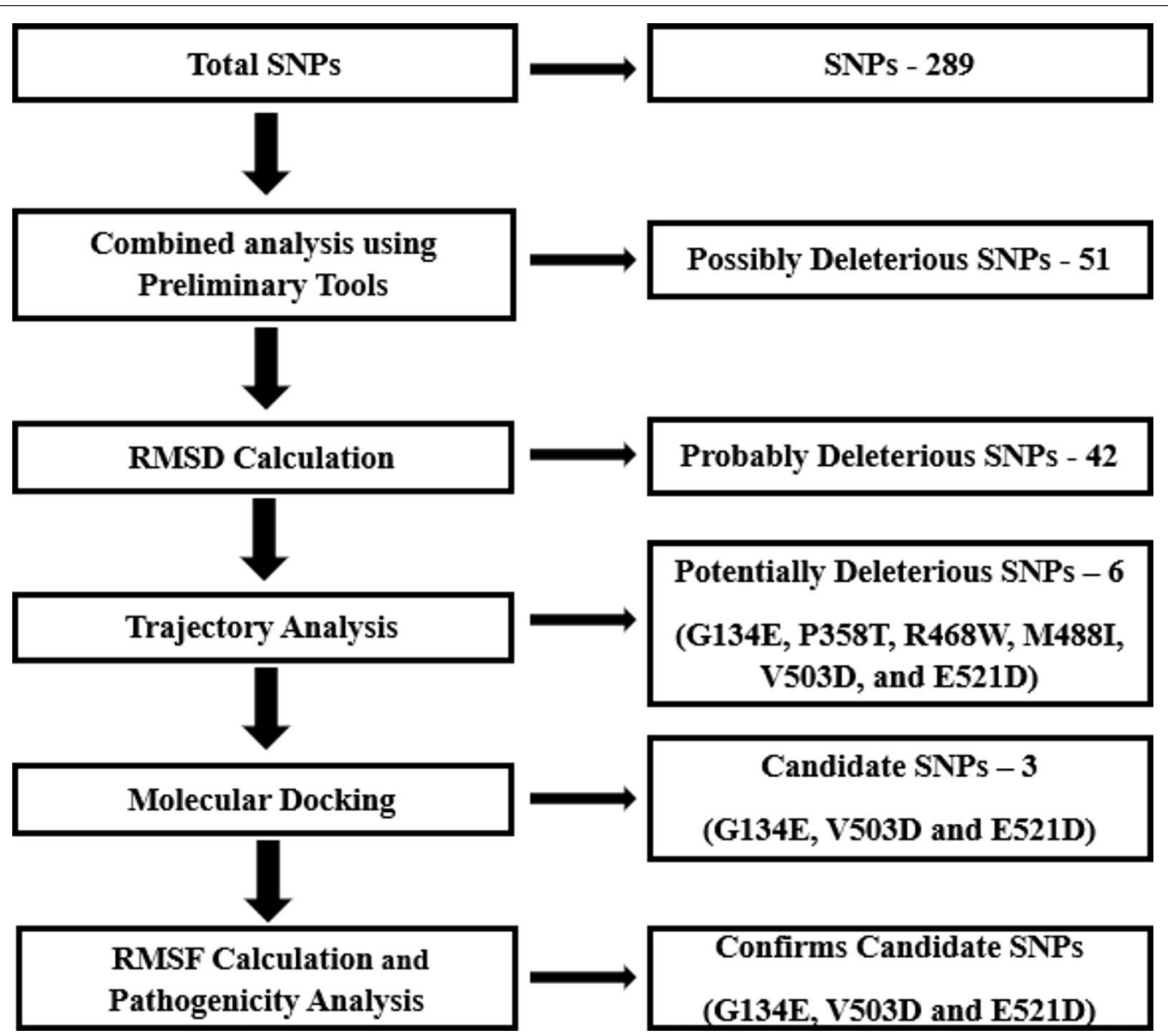

Fig. 2 Result summary of the entire work-flow 

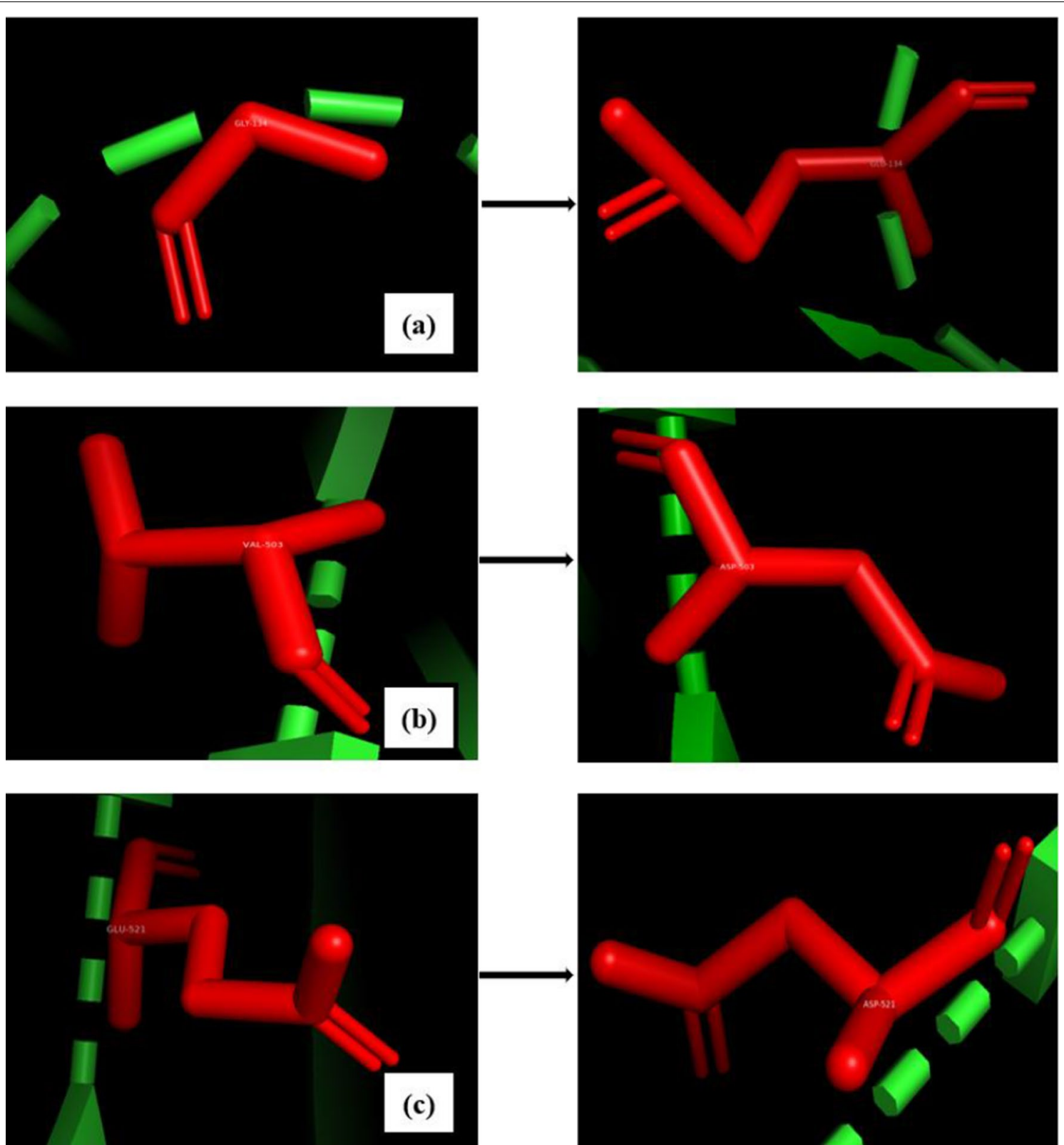

Fig. 3 Comparison of native (left) and mutant (right) structure using PyMol. a G134E, b V503D and c V521D

\section{RMSF calculation}

RMSF calculation done using the CABS-flex server, confirms G134E, V503D, and E521D as candidate ns-SNPs with variations in RMSF values (Table 7). Obtained result revealed that the values for mutants were lower when compared with native structure. This confirms the compressed nature of protein structure which in turn can affect the protein function [44].

\section{Effect of SNP on splicing}

Netgene 2 employs an artificial neural network that predicts the splice site location. The DNA sequence in FASTA format for native and all three identified candidate SNPs were given as inputs and splice sites were predicted. The results of the candidate SNPs splice site prediction were compared with the native prediction. As a result, we found that these SNPs do not interfere with the splicing mechanism of the IDS gene. 


\section{Pathogenicity analysis}

Pathogenicity analysis was performed using DUET web server. It is an online server used to analyse the pathogenic effects of missense mutations in proteins. This server provides the integrative result by combining two independent approaches, namely $\mathrm{mCSM}$ and SDM. The results of this analysis are provided in Table 8 , which predicts that all the three identified candidate SNPs will result in the destabilization of protein. This further supports the identification of G134E, V503D, and E521D as candidate SNPs.

\section{Conclusion}

Analysis using various insilico tools predicts the influence of ns-SNPs on the structure and function of protein IDS. Out of 289 ns-SNPs, G134E, V503D and E521D with SNP IDs-rs193302910, rs398123248, and rs1602725543, respectively, were predicted as candidate ns-SNPs. These ns-SNPs may alter the structure of the protein and interfere with the functions. The result summary of the complete workflow is provided in Fig. 2 and the structure of the mutant is compared with the native structure using PyMol [45] in Fig. 3. The presence of these ns-SNPs may inactivate the enzyme IDS and results in accumulation of GAGs, ultimately leading to Hunter syndrome. These ns-SNPs can be considered for clinical confirmatory studies for understanding the exact mechanism and pathology of mutations, before used for diagnostics.

\section{Acknowledgements}

The authors are thankful to CSIR-NEIST for providing an opportunity to carry out this work. The authors thank the Researchers Supporting Project Number (RSP-2021/293) for financial support and King Saud University, Riyadh, Saudi Arabia.

\section{Authors' contributions}

AS: methodology, investigation, resources, data curation, writing-original draft. YD: project administration. WHA-Q: funding acquisition. MK: project administration. JR: project administration. AC: project administration. ES: investigation, resources, writing-review and editing. APV: Investigation, resources. BMS: investigation, resources. MQ: investigation, resources. SRA: conceptualization, methodology, validation, writing-review and editing, supervision. All authors read and approved the final manuscript.

\section{Funding}

This study was funded by King Saud University (RSP-2021/293).

\section{Availability of data and materials}

All data analysed during the current study are available in the National Centre for Biotechnology Information (NCBI) repository, https://www.ncbi.nlm.nih. gov/.

\section{Declarations}

Ethics approval and consent to participate Not applicable.

\section{Consent for publication}

Not applicable.

\section{Competing interests}

The authors declare that they have no competing interests.

\section{Author details}

'Department of Biotechnology, Alagappa University, Karaikudi, Tamil Nadu, India. ${ }^{2}$ Department of Food Sciences and Nutrition, College of Food and Agriculture Sciences, King Saud University, Riyadh, Saudi Arabia. ${ }^{3}$ Department of Biotechnology, Vel Tech High-Tech Dr. Rangarajan Dr. Sakunthala Engineering College, Avadi-Vel Tech Road, Chennai, Tamil Nadu, India. ${ }^{4}$ Grassland and Forage Division, National Institute of Animal Science, Rural Development Administration, Cheonan, Jeollabuk-do, South Korea. ${ }^{5}$ Department of Biotechnology, Sree Sastha Institute of Engineering and Technology (Anna University), Chennai, Tamil Nadu, India. ${ }^{6}$ Department of Biotechnology, Kongunadu Arts and Science College, Coimbatore, Tamil Nadu, India. ${ }^{7}$ Department of Advanced Biochemistry, University of Madras, Chennai, Tamil Nadu, India. ${ }^{8}$ Department of Zoology, Punjabi University, Patiala, Punjab, India. ${ }^{9}$ Department of Agricultural Biotechnology, Tamil Nadu Agricultural University, Coimbatore, Tamil Nadu, India.

Received: 25 October 2021 Accepted: 16 February 2022

Published online: 06 March 2022

\section{References}

1. Yatziv S, Erickson RP, Epstein CJ (1977) Mild and severe Hunter syndrome (MPS II) within the same sibships. Clin Genet 11:319-326. https://doi.org/ 10.1111/j.1399-0004.1977.tb01323.x

2. Galvis J, González J, Uribe A, Velasco H (2015) Deep genotyping of the IDS Gene in Colombian Patients with Hunter Syndrome. JIMD Rep 19:101-109. https://doi.org/10.1007/8904_2014_376

3. Wilson PJ, Morris CP, Anson DS, Occhiodoro T, Bielicki J, Clements PR et al (1990) Hunter syndrome: isolation of an iduronate-2-sulfatase CDNA clone and analysis of patient DNA. Proc Natl Acad Sci U S A 87:85318535. https://doi.org/10.1073/pnas.87.21.8531

4. Shaklee P, Glaser J, Conrad H (1985) A sulfatase specific for glucuronic acid 2-sulfate residues in glycosaminoglycans. J Biol Chem 260:9146-9149

5. Demydchuk M, Hill C, Zhou A, Bunkoczi G, Stein PE, Marchesan D et al (2017) Insights into Hunter syndrome from the structure of iduronate2-sulfatase. Nat Commun 8:15786. https://doi.org/10.1038/ncomm s15786

6. Young ID, Harper PS (1982) Incidence of Hunter's syndrome. Hum Genet 60:391-392. https://doi.org/10.1007/BF00569230

7. Nelson J, Crowhurst J, Carey B, Greed L (2003) Incidence of the mucopolysaccharidoses in Western Australia. Am J Med Genet A 123A:310-313. https://doi.org/10.1002/ajmg.a.20314

8. Schumacher RG, Brzezinska R, Schulze-Frenking G, Pitz S (2008) Sonographic ocular findings in patients with mucopolysaccharidoses I, II and VI. Pediatr Radiol 38:543-550. https://doi.org/10.1007/s00247-008-0788-y

9. Martin R, Beck M, Eng C, Giugliani R, Harmatz P, Muñoz V, Muenzer J (2008) Recognition and diagnosis of mucopolysaccharidosis II (Hunter syndrome). Pediatrics 121:e377-e386. https://doi.org/10.1542/peds. 2007-1350

10. Sanjurjo-Crespo P (2007) Mucopolisacaridosis de tipo Il: aspectos clínicos (Clinical aspects of mucopolysaccharidosis type II). Rev Neurol. 44 Suppl 1:S3-S6 (Spanish)

11. Wraith JE, Scarpa M, Beck M, Bodamer OA, De Meirleir L, Guffon N et al (2008) Mucopolysaccharidosis type II (Hunter syndrome): a clinical review and recommendations for treatment in the era of enzyme replacement therapy. Eur J Pediatr 167:267-277. https://doi.org/10.1007/ s00431-007-0635-4

12. Giugliani R, Villarreal MLS, Valdez CAA, Hawilou AM, Guelbert N, Garzon LNC et al (2014) Guidelines for diagnosis and treatment of Hunter Syndrome for clinicians in Latin America. Genet Mol Biol 37:315-329. https:// doi.org/10.1590/s1415-47572014000300003 
13. Bradley L, Haddow H, Palomaki G (2017) Treatment of mucopolysaccharidosis type II (Hunter syndrome): results from a systematic evidence review. Genet Med 19:1187-1201. https://doi.org/10.1038/gim.2017.30

14. Sherry ST, Ward MH, Kholodov M, Baker J, Phan L, Smigielski EM, Sirotkin K (2001) dbSNP: the NCBI database of genetic variation. Nucl Acids Res 29:308-311. https://doi.org/10.1093/nar/29.1.308

15. Sim NL, Kumar P, Hu J, Henikoff S, Schneider G, Ng PC (2012) SIFT web server: predicting effects of amino acid substitutions on proteins. Nucl Acids Res 40(Web Server issue):W452-7. https://doi.org/10.1093/nar/ gks539

16. Adzhubei IA, Schmidt S, Peshkin L, Ramensky V, Gerasimova A, Bork P, Kondrashov A, Sunyaev SA (2010) Method and server for predicting damaging missense mutations. Nat Methods 7:248-249. https://doi.org/ 10.1038/nmeth0410-248

17. Mi H, Muruganujan A, Ebert D, Huang X, Thomas PD (2019) PANTHER version 14: more genomes, a new PANTHER GO-slim and improvements in enrichment analysis tools. Nucl Acids Res 47:D419-D426. https://doi.org/ 10.1093/nar/gky1038

18. Capriotti E, Fariselli P, Casadio R (2005) I-Mutant2.0: predicting stability changes upon mutation from the protein sequence or structure. Nucl Acids Res 33(Web Server issue):W306-W310. https://doi.org/10.1093/nar/ gki375.10.1093/nar/gki375

19. López-Ferrando V, Gazzo A, de la Cruz X, Orozco M, Gelpí JL (2017) PMut: a web-based tool for the annotation of pathological variants on proteins, 2017 update. Nucl Acids Res 45:W222-W228. https://doi.org/10.1093/ nar/gkx313

20. Waterhouse A, Bertoni M, Bienert S, Studer G, Tauriello G, Gumienny R, Heer FT, de Beer TAP, Rempfer C, Bordoli L, Lepore R, Schwede T (2018) SWISS-MODEL: homology modelling of protein structures and complexes. Nucl Acids Res 46:W296-303. https://doi.org/10.1093/nar/gky427

21. Laskowski RA, MacArthur MW, Moss DS, Thornton JM (1993) PROCHECK: a program to check the stereochemical quality of protein structures. J Appl Cryst 26:283-291. https://doi.org/10.1107/S0021889892009944

22. Wiederstein M, Sippl MJ (2007) ProSA-web: interactive web service for the recognition of errors in three-dimensional structures of proteins. Nucl Acids Res 35:W407-W410. https://doi.org/10.1093/nar/gkm290

23. Guex N, Peitsch MC (1997) SWISS-MODEL and the Swiss-PdbViewer: an environment for comparative protein modeling. Electrophoresis 18:27142723. https://doi.org/10.1002/elps.1150181505

24. Abraham MJ, Murtola T, Schulz R, Páll S, Smith JC, Hess B, Lindahl E (2015) GROMACS: High performance molecular simulations through multi-level parallelism from laptops to supercomputers. SoftwareX 1:19-25. https:// doi.org/10.1016/j.softx.2015.06.001

25. Coutsias EA, Seok C, Dill KA (2004) Using quaternions to calculate RMSD. J Comput Chem 25:1849-1857. https://doi.org/10.1002/jcc.20110

26. Jones DT (1999) Protein secondary structure prediction based on position-specific scoring matrices. J Mol Biol 292:195-202. https://doi. org/10.1006/jmbi.1999.3091

27. Magyar C, Gromiha MM, Pujadas G, Tusnády GE, Simon I (2005) SRide: a server for identifying stabilizing residues in proteins. Nucl Acids Res 33(Web Server issue):W303-W305. https://doi.org/10.1093/nar/gki409

28. Ashkenazy H, Abadi S, Martz E, Chay O, Mayrose I, Pupko T, Ben-Tal N (2016) ConSurf 2016: an improved methodology to estimate and visualize evolutionary conservation in macromolecules. Nucl Acids Res 44:W344-W350. https://doi.org/10.1093/nar/gkw408

29. Klausen MS, Jespersen MC, Nielsen H, Jensen KK, Jurtz VI, Sønderby CK, Sommer MOA, Winther O, Nielsen M, Petersen B, Marcatili P (2019) NetSurfP-2.0: improved prediction of protein structural features by integrated deep learning. Proteins Struct Funct Bioinform 87:520-7. https://doi.org/ 10.1002/prot.25674

30. de Brevern AG, Bornot A, Craveur P, Etchebest C, Gelly JC (2012) PredyFlexy: flexibility and local structure prediction from sequence. Nucl Acids Res 40(Web Server issue):W317-22. https://doi.org/10.1093/nar/gks482

31. Ittisoponpisan S, Islam SA, Khanna T, Alhuzimi E, David A, Sternberg MJE (2019) Can predicted protein 3D structures provide reliable insights into whether missense variants are disease associated? J Mol Biol 431:21972212. https://doi.org/10.1016/j.jmb.2019.04.009

32. Trott O, Olson AJ (2010) AutoDock Vina: improving the speed and accuracy of docking with a new scoring function, efficient optimization and multithreading. J Comput Chem 31:455-461. https://doi.org/10.1002/jcc. 21334
33. Wallace AC, Laskowski RA, Thornton JM (1995) LIGPLOT: a program to generate schematic diagrams of protein-ligand interactions. Prot Eng 8:127-134. https://doi.org/10.1093/protein/8.2.127

34. Kuriata A, Gierut AM, Oleniecki T, Ciemny MP, Kolinski A, Kurcinski M, Kmiecik S (2018) CABS-flex 2.0: a web server for fast simulations of flexibility of protein structures. Nucl Acids Res. 46:W338-43. https://doi.org/ 10.1093/nar/gky356

35. Brunak S, Engelbrecht J, Knudsen S (1991) Prediction of human mRNA donor and acceptor sites from the DNA sequence. J Mol Biol 220:49-65. https://doi.org/10.1016/0022-2836(91)90380-O

36. Pires DE, Ascher DB, Blundell TL (2014) DUET: a server for predicting effects of mutations on protein stability using an integrated computational approach. Nucl Acids Res 42(W1):W314-W319. https://doi.org/10.1093/nar/gku411

37. Meléndez-Aranda L, Jaloma-Cruz AR, Pastor N, Romero-Prado MMdeJ (2019) In silico analysis of missense mutations in exons 1-5 of the F9 gene that cause hemophilia B. BMC Bioinform 20:363. https://doi.org/10. 1186/s12859-019-2919-x

38. Pandey S, Dhusia K, Katara P, Singh S, Gautam B (2019) An in-silico analysis of deleterious single nucleotide polymorphisms and molecular dynamics simulation of disease linked mutations in genes responsible for neurodegenerative disorder. J Biomol Struct Dyn 1:22. https://doi.org/10. 1080/07391102.2019.1682047

39. AbdulAzeez S, Borgio JF (2016) In-silico computing of the most deleterious nsSNPs in HBA1 gene. PLOS ONE 11:e0147702. https://doi.org/10. 1371/journal.pone.0147702

40. Chandrasekaran G, Hwang EC, Kang TW, Kwon DD, Park K, Lee JJ, Lakshmanan VK (2017) In silico analysis of the deleterious nsSNPs (missense) in the homeobox domain of human HOXB13 gene responsible for hereditary prostate cancer. Chem Biol Drug Des 90:188-199. https://doi.org/10.1111/cbdd.12938

41. Manzoor MF, Ahmad N, Manzoor A, Kalsoom A (2017) Food based phytochemical luteolin their derivatives, sources and medicinal benefits. IJALS 3:195-207. https://doi.org/10.22573/spg.ijals.017.s12200084

42. Apweiler R, Attwood TK, Bairoch A, Bateman A, Birney E, Biswas M et al (2001) The InterPro database, an integrated documentation resource for protein families, domains and functional sites. Nucl Acids Res 29:37-40. https://doi.org/10.1093/nar/29.1.37

43. Sunkar S, Neeharika D (2020) CYP2R1 and CYP27A1 genes: An in silico approach to identify the deleterious mutations, impact on structure and their differential expression in disease conditions. Genomics 112:36773686. https://doi.org/10.1016/j.ygeno.2020.04.017

44. Pandey S, Dhusia K, Katara P, Singh S, Gautam B (2019) An in-silico analysis of deleterious single nucleotide polymorphisms and molecular dynamics simulation of disease linked mutations in genes responsible for neurodegenerative disorder. J Biomol Struct Dyn 38:4259-4272. https://doi.org/ 10.1080/07391102.2019.1682047

45. Yuan S, Chan HCS, Hu Z (2017) Using PyMOL as a platform for computational drug design. Wiley Interdiscip Rev Comput Mol Sci 7:e1298. https:// doi.org/10.1002/wcms.1298

\section{Publisher's Note}

Springer Nature remains neutral with regard to jurisdictional claims in published maps and institutional affiliations.

\section{Submit your manuscript to a SpringerOpen ${ }^{\odot}$ journal and benefit from:}

- Convenient online submission

- Rigorous peer review

- Open access: articles freely available online

- High visibility within the field

Retaining the copyright to your article

Submit your next manuscript at springeropen.com 